\title{
Continuous multiple injections at the Fermilab Main Injector
}

\author{
K. Y. Ng* \\ Fermi National Accelerator Laboratory, Mail Stop 220, P.O. Box 500, Batavia, Illinois 60540
}

(Received 1 March 2002; revised manuscript received 9 May 2002; published 24 June 2002)

\begin{abstract}
Instead of slip stacking, an alternate method of doubling the linear intensity of the Fermilab Main Injector is discussed. This method makes use of rf barriers to transfer 12 booster batches from the Fermilab Booster to the Main Injector in 12 consecutive booster cycles, totaling $800 \mathrm{~ms}$. After that, adiabatic capture of the beam into $53 \mathrm{MHz}$ buckets can be accomplished in about $10 \mathrm{~ms}$. Because the beam is debunched during the injection process and no rf voltage is required, the beam loading voltages in the rf cavities are small and can be eliminated by a combination of counterphasing and mechanical shorts.
\end{abstract}

DOI: 10.1103/PhysRevSTAB.5.061002

PACS numbers: 29.27.Ac

\section{INTRODUCTION}

In the Fermilab Run IIa, the Fermilab Main Injector is supposed to deliver one booster batch of $5.0 \times 10^{12}$ protons ( 84 bunches each containing $6 \times 10^{10}$ protons) to the target for the production of antiprotons in a $1.5 \mathrm{~s}$ acceleration cycle [1]. In the Run IIb upgrade [2], the number of protons delivered will be doubled. The method to accomplish this is through slip stacking two booster batches [3]. At the injection total energy of $E=8.938 \mathrm{GeV}$, the Main Injector has a circumference in time of $T_{0}=11.13 \mu \mathrm{s}$, which is exactly seven booster batches long. The acceleration cycle time in Run IIb will be increased to $2.0 \mathrm{~s}$. This cycle time should be long enough to load the Main Injector with six booster batches each of length $T_{b}=1.59 \mu \mathrm{s}$, slip stack one batch to be used for antiroton production, and extract the other five batches for the NuMI neutrino experiment. This cycle is sketched in Fig. 1.

Slip stacking had been studied extensively at the CERN Proton Synchrotron to double the number of protons per bunch for the production of antiprotons. However, due to the high loss and large increase in final longitudinal and horizontal emittances, the method had never been used in production runs [4]. The main disadvantage of this project is that it involves rf manipulations of intense beams at very low $\mathrm{rf}$ voltages resulting in a severe beam loading situation. At Fermilab, we plan on correcting the beam loading with direct rf feedback around the rf cavities in the Main Injector. Simulations show that very large loop gains are needed to remove the beam loading to a sufficient level [5]. The large loop gains, however, are associated with a number of stability issues.

An alternative way to double the density of the proton bunches is through the use of rf barrier waves. First, inject two booster batches into the Main Injector. Second, introduce two barriers at the ends of the two-batch region. Third, squeeze the barriers to compress the two-batch

*Electronic address: ng@fnal.gov region to a one-batch length resulting in doubling the longitudinal intensity. Fourth, capture the compressed doubledensity batch into $53 \mathrm{MHz}$ rf buckets. Finally, fill the next five booster lengths with one booster batch each. The result is a double-density batch in the first section for antiproton production followed by five single-batch sections for the NuMI neutrino experiment. This method of increasing beam density using barriers has been studied theoretically by Machida [6] and $\mathrm{Ng}$ [7], and experimentally at the Brookhaven Alternating Gradient Synchrotron [8]. However, this method has the disadvantage of being too time consuming. In order not to increase the longitudinal emittance of the beam, the bunch compression by barrier squeezing has to be adiabatic. The barrier movement speed $\dot{T}_{2}$ must be very much less than one-half the maximum phase-drifting speed of the particles with the largest fractional momentum offset [7], or

$$
\left|\dot{T}_{2}\right| \ll \frac{1}{2}|\eta \delta|,
$$

where $\eta$ is the slip factor and $\delta$ is the maximum fractional momentum half spread of the beam. Thus, for the compression of a booster-batch length $T_{b}$, the time required becomes

$$
\text { Barrier squeezing time } \gg \frac{2 T_{b}}{|\eta \delta|} \text {. }
$$

Using the Main Injector parameters in Table I, even with a maximum fractional momentum half spread of $\delta=3.39 \times 10^{-3}$, the barrier squeezing time must be much larger than $0.36 \mathrm{~s}$. This will lengthen the Main Injector acceleration cycle significantly and is therefore unacceptable.

Another method of doubling beam density by barriers was introduced by Griffin [9]. This is a momentum stacking method which does not require adiabatic bunch compression. As a result, injection from the Fermilab Booster can proceed batch by batch at the booster cycling rate, and all six batches can have their density doubled in 12 booster cycles. After that the beam will be captured adiabatically 


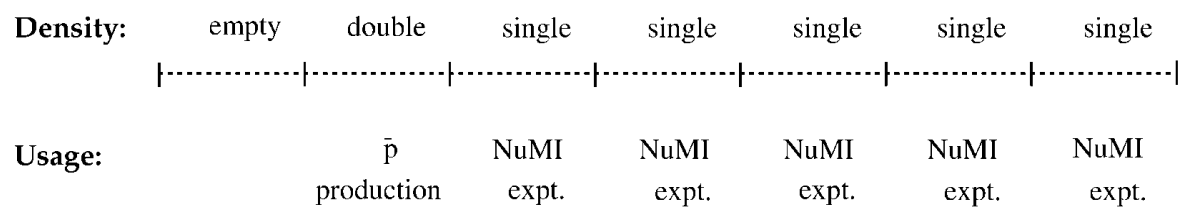

FIG. 1. The Main Injector in Run IIb accelerates five single-density booster batches for the NuMI neutrino experiment and one double-density booster batch for antiproton production. One booster-batch length is left empty for the kickers. At the injection energy of $E=8.939 \mathrm{GeV}$, one booster-batch length is $T_{b}=1.59 \mu \mathrm{s}$ and the revolution period of the Main Injector is $T_{0}=7 T_{b}=$ $11.13 \mu \mathrm{s}$.

TABLE I. Some injection parameters of the Fermilab Main Injector.

\begin{tabular}{lc}
\hline \hline Nominal total energy $E(\mathrm{GeV})$ & 8.9383 \\
Relativistic $\gamma / \beta$ & $9.326 / 0.99448$ \\
Transition gamma & 21.8 \\
Slip factor $\eta$ & -0.008915 \\
Revolution period $T_{0}(\mu s)$ & 11.1339 \\
Length of booster batch $T_{b}=T_{0} / 7(\mu \mathrm{s})$ & 1.5906 \\
Booster repetition rate $(\mathrm{Hz})$ & 15 \\
\hline \hline
\end{tabular}

into $53 \mathrm{MHz}$ buckets in about $10 \mathrm{~ms}$ before acceleration takes place. The whole accelerator cycle should be well within the proposed $2 \mathrm{~s}$. In this paper, we are going to examine this method in detail. Some simulations will be presented.

\section{THE INJECTION METHOD}

Let us start with a booster batch of protons of length $T_{b}$ and full fractional momentum spread $\Delta$. This batch, denoted by 1 in Fig. 2(a), is injected into the Main Injector at a negative momentum offset, so that the highest fractional momentum offset ${ }^{1}$ is $\delta_{i 1}$ and the lowest fractional momentum offset is $\delta_{i 2}=\delta_{i 1}-\Delta$.

The longitudinal position of the injection is chosen so that the right side of the batch just touches the left side of a square barrier (denoted by B) of width $T_{1}$ and magnitude $V$, which is moving to the left at the speed of $\dot{T}_{2}=$ $T_{b} /\left(2 T_{c}\right)$ where $T_{c}$ is the booster-cycle time. The momentum offset of the batch injection is chosen so that the proton of highest energy on the left side of the batch will drift to the right at the speed of $\frac{1}{2} T_{b}$ per booster cycle or $-\dot{T}_{2}$. Thus, after one booster cycle, another batch marked 2 in Fig. 2(b) can be injected again with its right edge touching the left side of the barrier. In other words, at every booster cycle, a new booster batch can be injected with the injection point moved half a booster-batch length to the left. The linear beam density can therefore be doubled.

The only parameter here is the size of the barrier, which is chosen so that after the booster batch emerges from the barrier, the highest and lowest energies of the batch be-

\footnotetext{
${ }^{1}$ We denote by the subscript 1 the particle at the upper right corner of the batch and 2 the particle at the lower right corner of the batch.
}

come symmetric about the nominal energy of the accelerator ring. This is necessary because we need to place another stationary barrier on the right side of the moving barrier in order to limit the longitudinal motion of the protons after passing through the moving barrier so as to guarantee empty spaces along the Main Injector for successive batch transfer from the Booster. It will be shown in the Appendix that the injection method described here depends just on the integrated size of the barrier and is independent of its shape. As a result, we denote the size of the barrier by $V T_{1}$ and a barrier of any shape can be used. However, for the ease of derivation, we continue to assume a rectangular barrier.

The mathematic determination of $V T_{1}$ is as follows. Let us start from the time when the proton at the upper right corner of the batch enters the barrier. After $n$ turns into the barrier, the fractional momentum offset is

$$
\delta_{n 1}=\delta_{i 1}+n \Delta \delta
$$

(a)

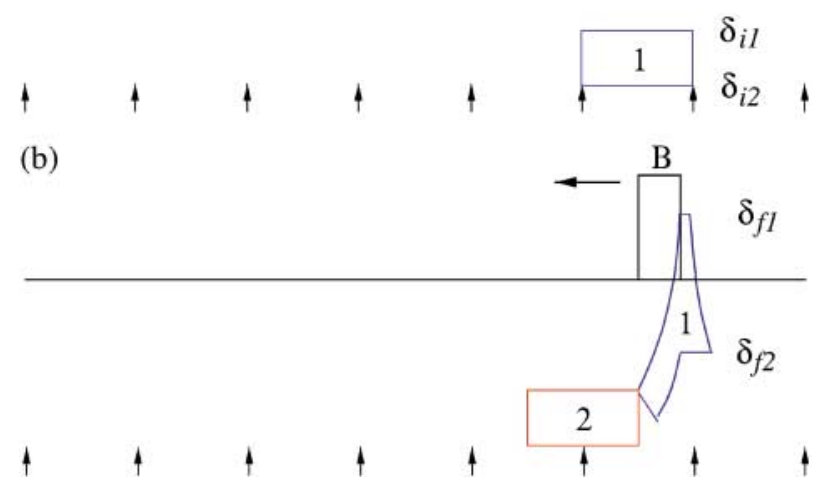

FIG. 2. (Color) (a) A booster batch with its head at the left (marked 1) is injected at negative momentum offset with the right edge just touching a left-moving barrier (marked B). The vertical arrows are spaced one booster-batch length $T_{b}$. (b) After a booster cycle, the barrier moves by half a booster-batch length while all particles in batch 1 drift past the left edge of the barrier. After exiting the barrier, the batch has maximum and minimum momenta, $\delta_{f 1}$ and $\delta_{f 2}$, symmetric about the onmomentum particles. A second booster batch (marked 2) is now injected. 
where

$$
\Delta \delta=\frac{e V}{\beta^{2} E}
$$

is the fractional momentum gained by the particle encountering the barrier per turn, $e$ is the proton charge, $E$ is the on-momentum energy of the protons in the Main Injector, $\beta c$ is the particle longitudinal velocity, and $c$ is the velocity of light. Suppose this proton exits the barrier in the $N_{1}$ turns. Its phase drift in time ${ }^{2}$ towards the right is

$$
\int_{0}^{N_{1}} \eta T_{0}\left(\delta_{i 1}+n \Delta \delta\right) d n,
$$

where $\eta$ is the slip factor and $T_{0}$ is the revolution period. Here, both $\eta$ and $\delta_{i 1}$ are negative. At this moment, the barrier has moved towards the left by the phase $N \dot{T}_{2} T_{0}$. Because the width of the barrier is $T_{1}$, we must have

$$
T_{1}-N_{1} \dot{T}_{2} T_{0}=\int_{0}^{N_{1}} \eta T_{0}\left(\delta_{i 1}+n \Delta \delta\right) d n
$$

The turn number $N_{1}$ therefore satisfies the quadratic equation

$$
N_{1}^{2}+2 N_{1} \frac{\delta_{i 1}-\dot{T}_{2} /|\eta|}{\Delta \delta}-\frac{2 T_{1}}{\eta T_{0} \Delta \delta}=0 .
$$

Noting that the Main Injector is below transition at injection, the solution is

$$
\begin{aligned}
N_{1}= & -\frac{\delta_{i 1}-\dot{T}_{2} /|\eta|}{\Delta \delta} \\
& -\sqrt{\left(\frac{\delta_{i 1}-\dot{T}_{2} /|\eta|}{\Delta \delta}\right)^{2}-\frac{2 T_{1}}{|\eta| T_{0} \Delta \delta}} .
\end{aligned}
$$

We see that since the first term on the right is positive, both \pm signs before the square root are allowed to arrive at a positive turn number $N_{1}$. However, when the barrier width goes to zero $\left(T_{1} \rightarrow 0\right)$, the number of turns to clear the barrier also goes to zero $\left(N_{1} \rightarrow 0\right)$. Since $\delta_{i 1}-\dot{T}_{2} /|\eta|<0$, this validates the choice of the negative sign. Substituting into Eq. (2.1), we obtain the final fractional momentum offset of the proton concerned,

$$
\begin{aligned}
\delta_{f 1} & =\delta_{i 1}+N_{1} \Delta \delta \\
& =\frac{\dot{T}_{2}}{|\eta|}-\sqrt{\left(\delta_{i 1}-\frac{\dot{T}_{2}}{|\eta|}\right)^{2}-\frac{2 T_{1} \Delta \delta}{|\eta| T_{0}}} .
\end{aligned}
$$

The final fractional momentum offset of the proton with the lowest energy is also given by Eq. (2.7) when the substitutions of the subscripts $i 1 \rightarrow i 2$ and $f 1 \rightarrow f 2$ are made. In order for final momentum spreads of the protons

\footnotetext{
${ }^{2}$ We denote the phase drift as time arrival behind of some on-momentum particle. Because the Main Injector at injection is below transition or $\eta<0$, this phase drift or arrival time is positive for a negative-momentum-offset particle.
}

to be symmetric about the nominal energy $E$, we require $\delta_{f 1}=-\delta_{f 2}$, or

$$
\begin{aligned}
\frac{\dot{T}_{2}}{|\eta|}-\sqrt{\left(\delta_{i 1}-\frac{\dot{T}_{2}}{|\eta|}\right)^{2}-\frac{2 T_{1} \Delta \delta}{|\eta| T_{0}}} \\
=-\left[\frac{\dot{T}_{2}}{|\eta|}-\sqrt{\left(\delta_{i 2}-\frac{\dot{T}_{2}}{|\eta|}\right)^{2}-\frac{2 T_{1} \Delta \delta}{|\eta| T_{0}}}\right] .
\end{aligned}
$$

Now we substitute for $\delta_{i 1}=-\dot{T}_{2} /|\eta|, \delta_{i 2}=\delta_{i 1}-\Delta$, and express everything in terms of $2 \dot{T}_{2} /|\eta|$. The above equation simplifies to ${ }^{3}$

$$
1-\sqrt{1-A}=\sqrt{(\bar{\Delta}+1)^{2}-A},
$$

where $\Delta=\bar{\Delta}\left(2 \dot{T}_{2} /|\eta|\right)$,

$$
A=\frac{2 T_{1} \Delta \delta}{|\eta| T_{0}}\left(\frac{\eta}{2 \dot{T}_{2}}\right)^{2}
$$

which is proportional to $V T_{1}$, the size of the barrier. We can readily obtain

$$
A=\frac{1}{4}(1+\bar{\Delta})^{2}(\bar{\Delta}+3)(\bar{\Delta}-1),
$$

or

$$
\begin{aligned}
V T_{1}= & \frac{|\eta|^{3} \beta^{2} E T_{0}}{32 e \dot{T}_{2}^{2}}\left(2 \delta_{i 1}-\Delta\right)^{2}\left(2 \delta_{i 1}-\Delta-\frac{4 \dot{T}_{2}}{|\eta|}\right) \\
& \times\left(\Delta-\frac{2 \dot{T}_{2}}{|\eta|}\right) .
\end{aligned}
$$

\section{CRITICAL MOMENT}

Obviously, a larger initial fractional momentum spread $\Delta$ of the beam will lead to a larger final fractional momentum spread. However, when the initial fractional momentum spread is large enough, protons with the highest momentum can acquire so much energy from the moving barrier that their drifting speeds to the left exceed the speed of the moving barrier. The result is that these particles will not be able to emerge from the moving barrier and this injection method fails. This happens when

$$
\dot{T}_{2}<|\eta| \delta_{f 1},
$$

where $\delta_{f 1}$ is given by Eq. (2.7), or the expression under the square root of Eq. (2.7) becomes negative. This gives us the critical size of the moving barrier

$$
\left(V T_{1}\right)_{c}=\frac{|\eta| \beta^{2} E T_{0}}{2 e}\left(\frac{2 \dot{T}_{2}}{|\eta|}\right)^{2} .
$$

The same can be inferred by squaring Eq. (2.9) to give

$$
\sqrt{1-A}=1-\frac{(1+\bar{\Delta})^{2}}{2}
$$

and notice that the left side is positive semidefinite, while the right side can be negative. This leads to the critical

\footnotetext{
${ }^{3}$ Every normalized variable carries a bar.
} 
value of $A=1$ which is the expression given by Eq. (3.2) and the corresponding maximum normalized $\bar{\Delta}_{c}=\sqrt{2}-$ 1 . Thus the maximum allowable initial full fractional momentum spread of the beam is

$$
\Delta_{c}=(\sqrt{2}-1) \frac{2 \dot{T}_{2}}{|\eta|} .
$$

For the Main Injector, these critical numbers are $\left(V T_{1}\right)_{c}=3.1421 \mathrm{kV} \mu \mathrm{s}$ and $\Delta_{c}=0.001109$. The critical half energy spread of the batch is therefore $\Delta E_{1 / 2 c}=$ $\Delta_{c} \beta^{2} E / 2=4.900 \mathrm{MeV}$.

\section{GENERALIZED METHOD}

In order to incorporate initial full momentum spread of the beam larger than the critical value of $\Delta_{c}=0.001109$, the obvious way is to let the barrier move to the left at a higher speed. Let the barrier move to the left at the speed $x T_{b}$ for each booster cycle of $T_{c}=1 / 15 \mathrm{~s}$ so that $\dot{T}_{2}=$ $x T_{b} / T_{c}\left(x=\frac{1}{2}\right.$ in Secs. II and III). To ensure injection for each booster cycle, an injected batch must clear the left edge of the moving barrier in a booster cycle. Therefore, protons in the batch with top energy must drift to the right at the speed of $y T_{b} / T_{c}=y \dot{T}_{2} / x$, with $x+y=1$. In other words, these top-energy protons at injection must have fractional momentum offset given by

$$
\delta_{i 1}=-\frac{y \dot{T}_{2}}{x|\eta|} .
$$

The derivation proceeds in the same way as in Sec. II. These protons have final fractional momentum offset given by Eq. (2.7) and the equation for the determination of $V T_{1}$ is again given by Eq. (2.8). Substituting for the value of $\delta_{i 1}$ given by Eq. (4.1) and normalizing everything with respect to $2 T_{2} /|\eta|$, we obtain, instead of Eq. (2.9),

$$
1-\sqrt{\frac{1}{4 x^{2}}-A}=\sqrt{\left(\frac{1}{2 x}+\bar{\Delta}\right)^{2}-A},
$$

where $A$ is still given by Eq. (2.10). Squaring, we get

$$
2 \sqrt{\frac{1}{4 x^{2}}-A}=1-\frac{\bar{\Delta}}{x}-\bar{\Delta}^{2} .
$$

To ensure that the right side will not become negative, we obtain the critical condition

$$
\bar{\Delta}<\sqrt{\frac{1}{4 x^{2}}+1}-\frac{1}{2 x} .
$$

Putting back the normalization factor $2 \dot{T}_{2} /|\eta|=$ $2 x T_{b} /\left(|\eta| T_{c}\right)$, we obtain the critical full fractional momentum spread of the beam

$$
\Delta_{c}=\left(\sqrt{x^{2}+\frac{1}{4}}-\frac{1}{2}\right) \frac{2 T_{b}}{|\eta| T_{c}} .
$$

Conversely, given a full fractional momentum spread $\Delta$, the speed of the moving barrier must be faster than $x T_{b} / T_{c}$ where

$$
x=\sqrt{\frac{|\eta| T_{c} \Delta}{2 T_{b}}\left(\frac{|\eta| T_{c} \Delta}{2 T_{b}}+1\right)} .
$$

For example, given the barrier moving rate $x$, and given an initial full momentum spread $\Delta<\Delta_{c}$, what is the required $V T_{1}$ for the moving barrier? This can be obtained by continuing the solution of Eq. (4.3). The final answer is

$$
\begin{aligned}
V T_{1}= & \frac{T_{0} T_{b}^{2} \beta^{2} E}{2|\eta| T_{c}^{2}} 4 x^{2} A=\frac{T_{0} T_{b}^{2} \beta^{2} E}{2|\eta| T_{c}^{2}}\left[1-\bar{\Delta}^{2}\right] \\
& \times\left[(1+x \bar{\Delta})^{2}-x^{2}\right] .
\end{aligned}
$$

The critical size of the moving barrier can be obtained easily from Eq. (4.3):

$$
A=\frac{1}{4 x^{2}}
$$

Since $A$ is given by

$$
A=\frac{2 T_{1} \Delta \delta}{|\eta| T_{0}}\left(\frac{|\eta|}{2 \dot{T}_{2}}\right)^{2}=\frac{2 T_{1} \Delta \delta}{|\eta| T_{0}}\left(\frac{|\eta| T_{c}}{T_{b}}\right)^{2} \frac{1}{4 x^{2}},
$$

the critical size of the moving barrier is independent of $x$ and remains given by

$$
\left(V T_{1}\right)_{c}=\frac{T_{0} T_{b}^{2} \beta^{2} E}{2 e|\eta| T_{c}^{2}},
$$

which is $3.1421 \mathrm{kV} \mu \mathrm{s}$ for the Main Injector.

At the critical condition, the injection energy offset $\Delta E_{i 1}=\delta_{i 1} \beta^{2} E$ and final half energy spread $\Delta E_{f 1}=\delta_{f 1} \beta^{2} E$ are plotted in Fig. 3 as functions of initial half energy spread $\Delta E_{1 / 2 i}$. As the initial half energy spread increases, the critical speed of the barrier increases. The top-energy particles will be drifting at the same speed as the barrier and therefore the final half energy spread increases. On the other hand, the drifting of the batch at injection decreases, so does the initial energy offset at injection. For a given barrier speed

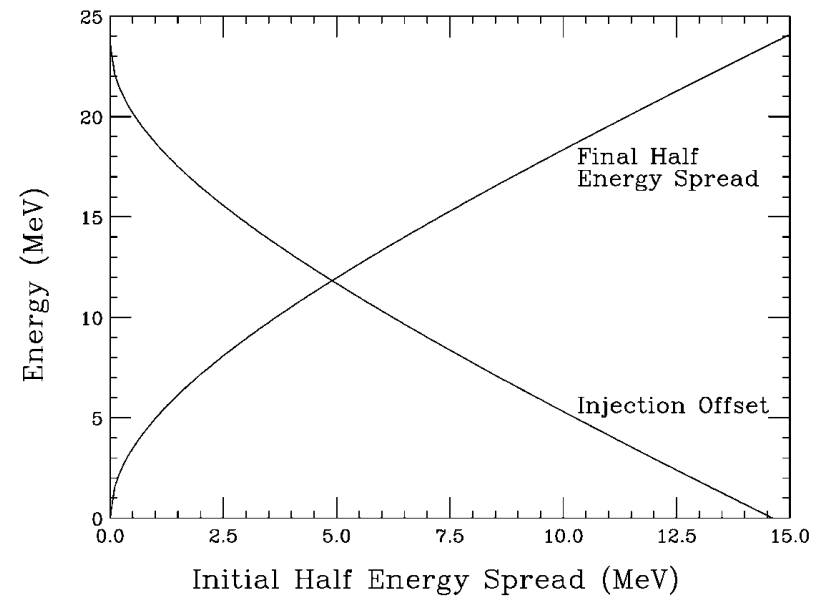

FIG. 3. At the critical condition, the initial energy offset at injection $\Delta E_{i 1}=\delta_{i 1} \beta^{2} E$ and final half energy spread $\Delta E_{f 1}=$ $\delta_{f 1} \beta^{2} E$ versus the initial half energy spread of the booster batch. 


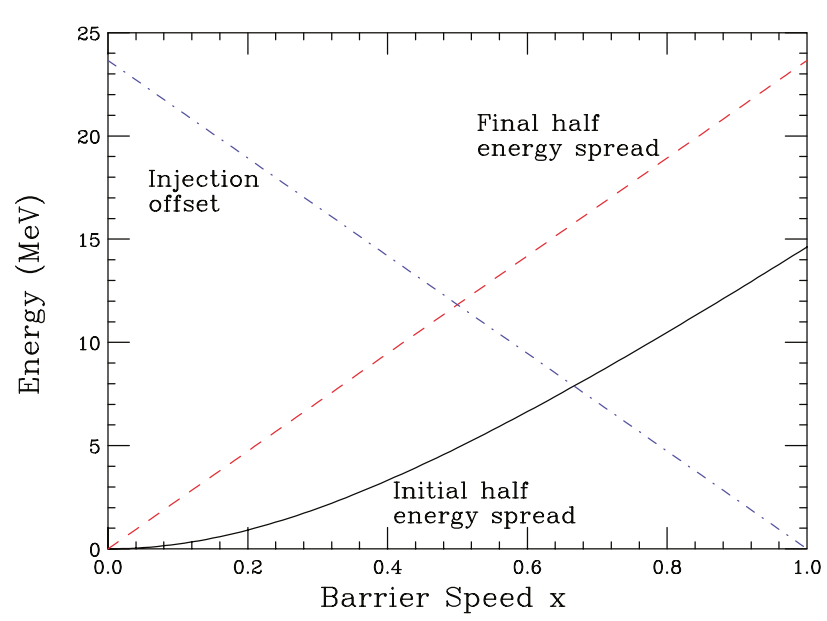

FIG. 4. (Color) Given a barrier speed, the plot shows the critical initial half energy spread of the booster batch $\Delta E_{1 / 2 i}$, the initial energy offset $\Delta E_{i 1}$, and the final half energy offset $\Delta E_{f 1}$.

$\dot{T}_{2}=x T_{b} / T_{c}$, the critical injection energy offset $\Delta E_{i 1}$, critical initial half energy spread $\Delta E_{1 / 2 i}$, and critical final half energy spread $\Delta E_{f 1}$ can be looked up in Fig. 4. Thus, if the initial half energy spread $\Delta E_{1 / 2 i}$ is very small, we can perform the injection with a very slow speed for the moving barrier. This allows us to inject at every $x T_{b}$ along the accelerator ring and we can obtain $x^{-1}$-fold stacking in the momentum space. The final energy spread will therefore increase by $x^{-1}$ fold.

\section{EMITTANCE AND LINEAR DENSITY}

When the injection barrier is moving to the left at $x T_{b}$ per booster cycle, the position of injection along the accelerator ring slips to the left by $x T_{b}$ for each injection or for each booster cycle. This means that we can inject in the length $x T_{b}$ a whole batch of length $T_{b}$. Or we can inject in the length $T_{b}, 1 / x$ batches. Therefore the ratio of emittance by this method and the original emittance is

$$
r_{e}=\frac{\delta_{f 1} \times 1}{\frac{\Delta}{2} \times \frac{1}{x}} .
$$

Here, we assume the circumference of the accelerator ring is infinitely long so that injection can be continued forever. The final momentum offset $\delta_{f 1}$ is still given by Eq. (2.7) with $\delta_{i 1}=-y \dot{T}_{2} /(x|\eta|)$. Normalizing with respect to $2 \dot{T}_{2} /|\eta|$, we obtain

$$
\bar{\delta}_{f 1}=\frac{1}{2}-\sqrt{\frac{1}{4 x^{2}}-A} .
$$

With the help of Eq. (4.3), this gives

$$
\bar{\delta}_{f 1}=\frac{\bar{\Delta}}{2 x}(1+x \bar{\Delta}) .
$$

The emittance-growth ratio becomes

$$
r_{e}=1+x \bar{\Delta}=1+\frac{|\eta| T_{c} \Delta}{2 T_{b}} .
$$

It is interesting to see that this emittance growth is independent of $x$ or the speed at which the barrier moves; but it depends strongly on the initial momentum spread of the beam. The increase in emittance is evident in the lower plot of Fig. 2. We see that although the top-right corner of a batch is always connected to the top-left corner of a following batch, there is always a gap between the bottomright corner of a batch and the bottom-left corner of the following batch. This gap constitutes the increase in emittance because it will produce a triangular space between two consecutive batches after they exit the moving barrier (see Figs. 6-8).

Another important number is the final linear density. When the barrier is moving to the left at the speed of $x T_{b}$ per booster cycle, we can inject a batch at every length $x T_{b}$ rather than at every $T_{b}$ without the barrier. Thus, the ratio of linear density with barrier to the linear density without barrier is

$$
r_{d}=\frac{1}{x}
$$

For example, the linear density is doubled when $x=\frac{1}{2}$. For this reason, we should choose the lowest barrier moving speed allowable by the initial momentum spread of the batch. In other words, given $\Delta$, we should stick to the critical $x$ given by Eq. (4.6) and the critical size $\left(V T_{1}\right)_{c}$ for the barrier given by Eq. (4.10).

Given an initial half energy spread of the booster batch, the slowest speed $\dot{T}_{2}=x T_{b} / T_{c}$ of the moving barrier and the emittance increase $r_{e}-1$ are plotted in Fig. 5. We see that in order to keep the barrier speed at $\dot{T}_{2}=T_{b} /\left(2 T_{c}\right)$ or $x=\frac{1}{2}$, the half energy spread of the booster batch should not exceed $\Delta E_{1 / 2 i}=4.90 \mathrm{MeV}$. We also see that the longitudinal emittance increases linearly with $\Delta E_{1 / 2 i}$ as indicated by Eq. (5.4).

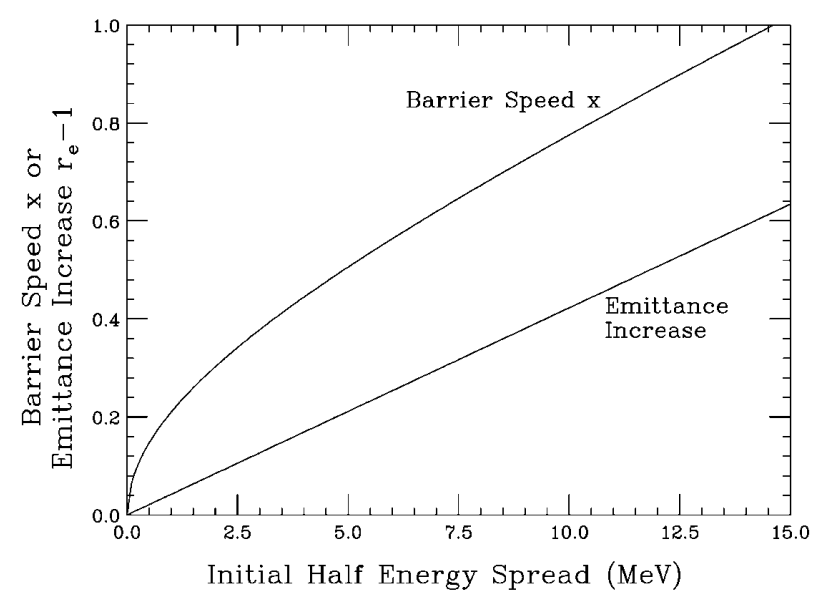

FIG. 5. The slowest or critical speed of the moving barrier and the longitudinal emittance increase versus the half energy spread of the booster batch. 


\section{APPLICATION TO MAIN INJECTOR}

At the extraction of the Fermilab Booster, a booster bunch has a bunch area of $\mathcal{A}=0.10 \mathrm{eV} \mathrm{s}$. Since there are $h_{B}=84$ bunches at $53 \mathrm{MHz}$ rf in the batch, if they are debunched adiabatically, the half energy spread is only

$$
\Delta E=\frac{h_{B} \mathcal{A}}{T_{b}}=2.64 \mathrm{MeV}
$$

However, time-consuming adiabatic debunching in the Booster is not possible because the Booster is a $15 \mathrm{~Hz}$ rapid-cycling machine. As a result, the rf voltage $V_{\text {rf }}$ must be lowered gradually after transition crossing to a small value near extraction. This process can also encounter problems, because the accelerating bucket will become smaller and beam loss can occur.

The half energy spread of a booster bunch is

$$
\Delta E=\sqrt{\frac{\omega_{s} \mathcal{A} \beta^{2} E}{\pi \eta}},
$$

where

$$
\omega_{s}=\omega_{0} \sqrt{\frac{h|\eta| V_{\mathrm{rf}}}{2 \pi \beta^{2} E}}
$$

is the angular synchrotron frequency and $\omega_{0} /(2 \pi)=$ $1 / T_{b}$ is the Booster revolution frequency. At extraction, the energy $E$ of a particle and its Lorentz factors are the same as those for the Main Injector at injection and are listed in Table I. The Booster has a transition gamma of $\gamma_{t}=5.6$; the slip factor at extraction is therefore $\eta=0.02087$. Thus if the rf voltage can be lowered to $V_{\mathrm{rf}}=10 \mathrm{kV}$, the half energy spread of the bunch becomes $\Delta E=5.47 \mathrm{MeV}$. This is still larger than the critical half energy spread of $\Delta E_{1 / 2 c}=4.900 \mathrm{MeV}$, if we would like the barrier to move at the speed given by $x=\frac{1}{2}$.

Another method of reducing the half energy spread is to introduce higher harmonic cavities into the Booster rf system so that the bunch can be made more rectangularlike. The rf wave becomes

$$
V(\theta)=V_{\mathrm{rf}}[\sin \theta-r \sin m \theta],
$$

where $\theta$ is the rf phase, $m$ the higher harmonic multiple, and $-r V_{\mathrm{rf}}$ the voltage of the higher harmonic cavity. If we let $r=1 / m$, the rf potential becomes quartic at small amplitude. The bunch is therefore lengthened with its energy spread lowered. For a $\mathcal{A}=0.11 \mathrm{eV}$ s bunch at $V_{\mathrm{rf}}=9.725 \mathrm{kV}$, the half energy spread is reduced [10] from $\Delta E=5.43 \mathrm{MeV}$ to $5.0 \mathrm{MeV}$ with the introduction of a $m=3$ higher harmonic cavity. For a $\mathcal{A}=0.10 \mathrm{eV} \mathrm{s}$ bunch at $V_{\mathrm{rf}}=4.8 \mathrm{kV}$, the half energy spread is reduced from $\Delta E=4.56 \mathrm{MeV}$ to $3.7 \mathrm{MeV}$ with the introduction of a $m=2$ higher harmonic cavity.

The momentum spread of the booster bunch can also be reduced in the Booster before extraction using a bunch rotation by one-quarter of a synchrotron oscillation. At this moment, longitudinal coupled-bunch instabilities are observed near extraction. Bunch rotation of an oscillating bunch will not necessarily produce a bunch of smaller momentum spread. Thus, the coupled-bunch instabilities must be cured by either deQing the offensive driving higherorder modes inside the booster cavities and/or repairing the longitudinal damper which has not been working properly at the present. We are confident that the half energy spread of a booster batch can be made below the critical $\Delta E_{1 / 2 c}=4.900 \mathrm{MeV}$ before extraction.

\section{A. Simulations}

We perform simulations of this injection method from the Booster to the Main Injector with the intention of doubling the linear particle density. Thus, we require the barrier movement of $\frac{1}{2} T_{b}$ per booster cycle or $x=\frac{1}{2}$, or $\dot{T}_{2}=$ $T_{b} /\left(2 T_{c}\right)=1.193 \times 10^{-5}$. The half energy spread of the booster batch is assumed to be $\Delta E_{1 / 2 i}=4.900 \mathrm{MeV}$ initially, exactly the critical value, for such barrier moving speed. Particles with the highest energy are injected with the offset of $\Delta E_{i 1}=-\dot{T}_{2} \beta^{2} E /|\eta|=-11.8285 \mathrm{MeV}$ so that, before entering the moving barrier, they drift at the same speed as the moving barrier but in the opposite direction. The moving barrier is at its critical value of $V T_{1}=3.1421 \mathrm{kV} \mu \mathrm{s}$ as given by Eq. (4.10).

From this information, particles with the lowest energy are injected with offset $\Delta E_{i 2}=\Delta E_{i 1}-2 \Delta E_{1 / 2 i}=$ $-21.6276 \mathrm{MeV}$. After exiting the moving barrier, the final energy offsets of particles with the highest and lowest energies are, respectively, $\Delta E_{f 1}=-\Delta E_{i 1}=11.8285 \mathrm{MeV}$ and $\Delta E_{f 2}=-\Delta E_{f 1}=-11.8285 \mathrm{MeV}$. These are tabulated in Table II. Thus, the energy spread has been increased by the factor $\Delta E_{f 1} / \Delta E_{1 / 2 i}=11.8285 / 4.8995=$ 2.419 and half of this, that is 1.209 , is the ratio of the final longitudinal emittance to the initial longitudinal emittance.

It is advisable to have the barrier voltage as high as possible, so that its width can be made narrow to allow for more space for injection. We choose $T_{1}=1.0 \mu \mathrm{s}$ so that $V=3.1421 \mathrm{kV}$. We start by placing this barrier with its left edge at a position which we denote by $6.5 T_{b}$ along the Main Injector as illustrated in the top plot of Fig. 6, where we also see the first batch injected with energy offset at the position between $5.5 T_{b}$ and $6.5 T_{b}$. Another stationary barrier is placed with its right edge at the $7.0 T_{b}$ point of the ring to block particles with negative energies so that their energy becomes positive after reflection and they drift to the left so as to ensure space for new injections. From the Hamiltonian in Eq. (A.2) or Eq. (A.5), the minimum size of this reflection barrier is

$$
V T_{1}=\frac{|\eta| T_{0} \Delta E_{f 1}^{2}}{2 \beta^{2} E}=0.7855 \mathrm{kV} \mu \mathrm{s} .
$$

To ensure that the barrier will catch all the right-drifting particles, we choose $V T_{1}=0.8 \mathrm{kV} \mu \mathrm{s}$. We further choose its width as $T_{1}=0.25 \mu$ s so that $V=3.2 \mathrm{kV}$, and the barrier is positioned between $6.75 T_{b}$ and $7.0 T_{b}$. The stationary barrier is represented by a red-filled box while the 
TABLE II. Some parameters in the simulations of this injection method from the Fermilab Booster to the Main Injector.

\begin{tabular}{lc}
\hline \hline$\dot{T}_{2}=T_{b} / T_{c}$ & $1.193 \times 10^{-5}$ \\
Half energy spread $\Delta E_{1 / 2 i}(\mathrm{MeV})$ & 4.900 \\
Injection energy offset: top of batch $\Delta E_{i 1}(\mathrm{MeV})$ & -11.8285 \\
$\quad$ bottom of batch $\Delta E_{i 2}(\mathrm{MeV})$ & -21.6276 \\
Final half energy spread $\Delta E_{f 1, f 2}(\mathrm{MeV})$ & \pm 11.8285 \\
Moving barrier $V T_{1}(\mathrm{kV} \mu \mathrm{s})$ & 3.142 \\
Minimum reflection barrier $V T_{1}(\mathrm{kV} \mu \mathrm{s})$ & 0.7855 \\
\hline \hline
\end{tabular}

moving barrier is represented by an unfilled box with an arrow inside pointing to the direction of its motion. After one booster cycle, we see in the bottom plot of Fig. 6 that all the particles of the first batch go past the left edge of
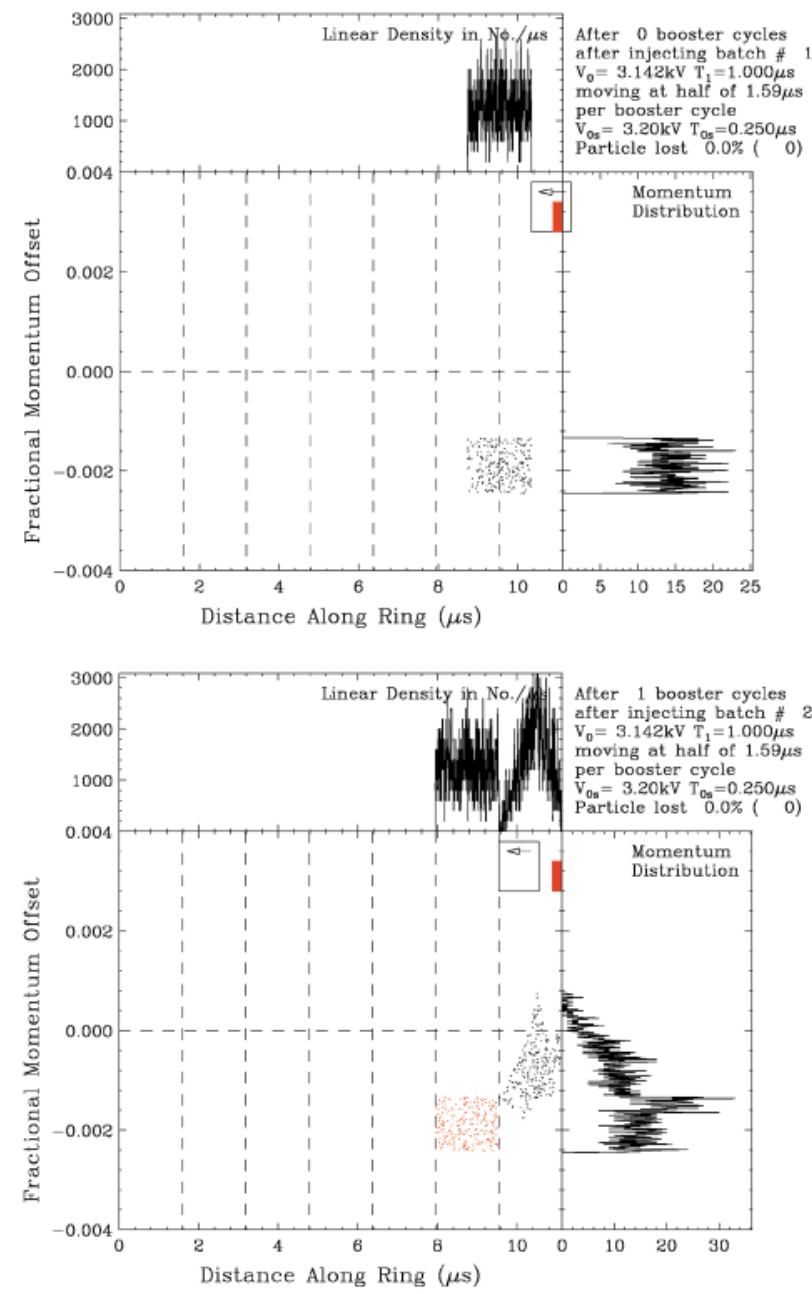

FIG. 6. (Color) Top: First batch (black) is injected between $5.5 T_{b}$ and $6.5 T_{b}$ with the right side touching the left side of the moving barrier, illustrated as an unfilled box with an arrow. Bottom: After one booster cycle, all particles in the first batch have just cleared the left side of the moving barrier, which is at $6.0 T_{b}$. The second batch (red) is now injected between $5.0 T_{b}$ and $6.0 T_{b}$. The moving barrier has a width of $T_{1}=1.0 \mu \mathrm{s}$ and a strength of $V=3.1421 \mathrm{kV}$. Vertical dashed lines are spaced at $T_{b}$. the moving barrier and some are reflected by the stationary barrier. At this moment, a second batch is injected in the region between $5.0 T_{b}$ and $6.0 T_{b}$. We alternate the color of successive batches so that their trajectories can be followed easily. The six vertical dashed lines divide the
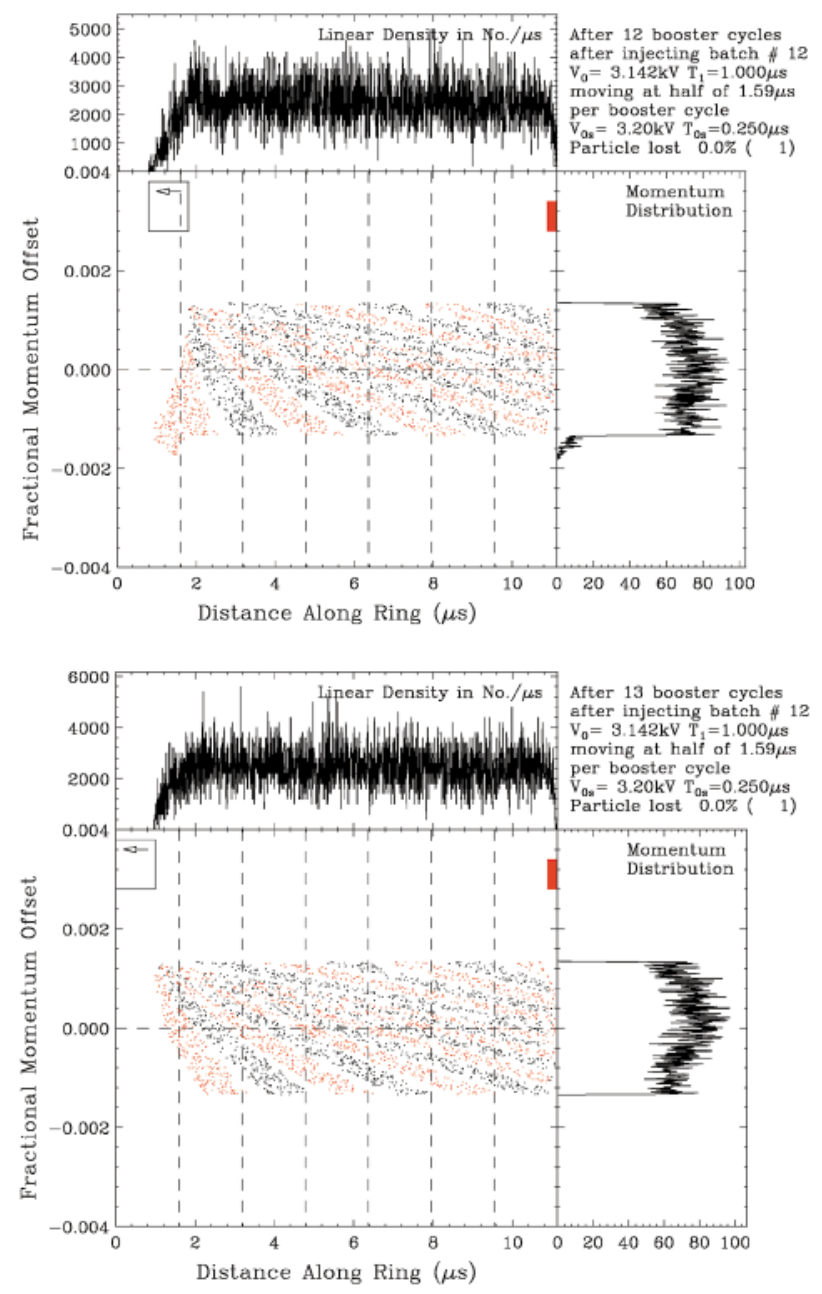

FIG. 7. (Color) Continuing the injection from Fig. 6. Top: One booster cycle after the injection of the 12th batch between $0.0 T_{b}$ and $1.0 T_{b}$, or 12 booster cycles after the injection of the first batch. Bottom: Another booster cycle later or 13 booster cycles after the injection of the first batch. All particles are within the designated final momentum spread and adiabatic capture can begin. 
circumference of the Main Injector into seven regions each of which is of length $T_{b}$ or one booster-batch long. Two thousand macroparticles are used to simulate each booster batch.

Successive batch injection continues. The situation of one booster cycle after the injection of the 12th batch between $0.0 T_{b}$ and $1.0 T_{b}$, or 12 booster cycles after the injection of the first batch, is shown in the top plot of Fig. 7. We need to wait until another booster cycle later, the bottom plot, to allow all the particles to drift inside the designated momentum spread. This is now the time to perform adiabatic capturing and acceleration. Thus an injection time of 13 booster cycles or $0.87 \mathrm{~s}$ will be required. We see empty spaces between successive batches in both plots of Fig. 7, indicating an increase in longitudinal emittance. The ratio of final emittance to initial emittance will be slightly larger than $r_{e}=1.209$ given by Eq. (5.4) because of the
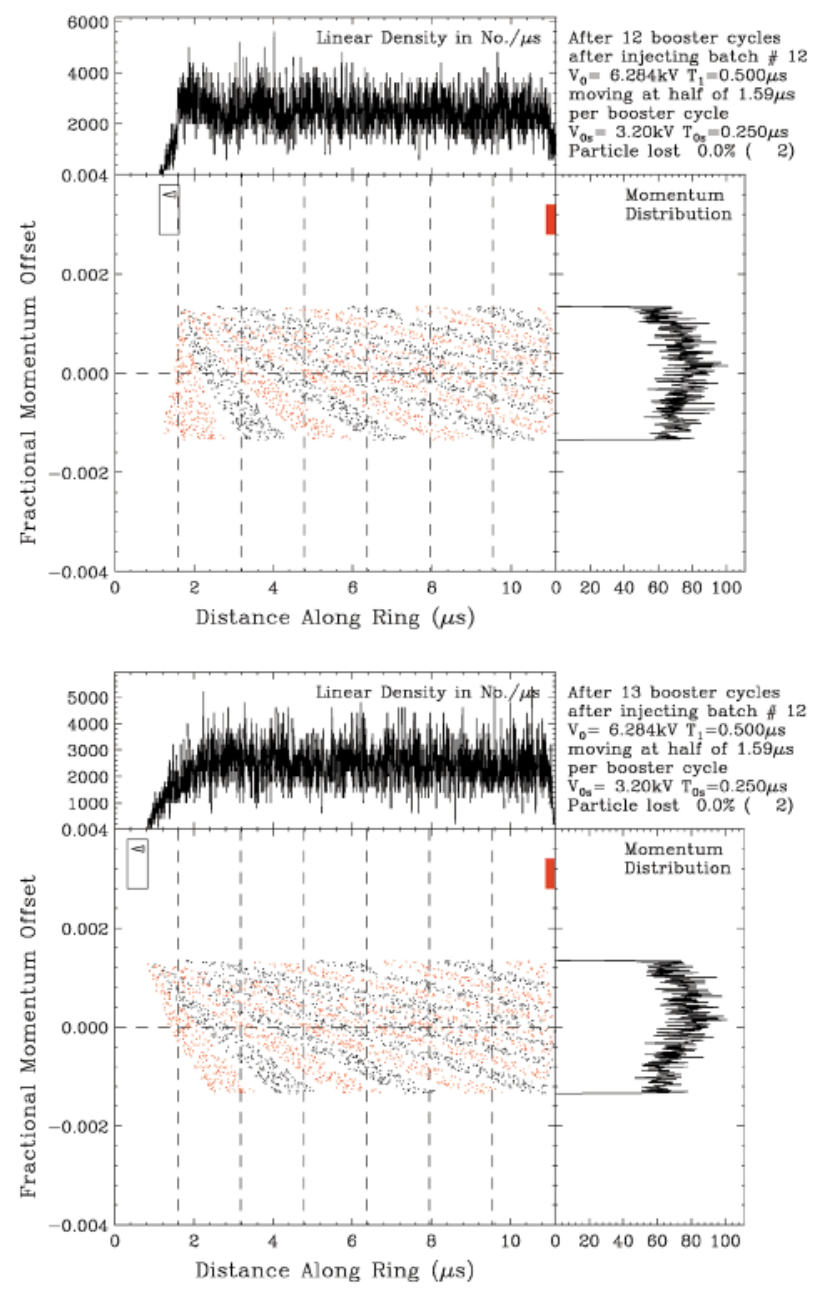

FIG. 8. (Color) Same as Fig. 7, but with the width of the moving barrier reduced to $0.5 \mu \mathrm{s}$ and strength increased to $6.2842 \mathrm{kV}$. At injection of the first batch, the left side of the barrier was placed at $6.7 T_{b}$. Top: One booster cycle after the injection of the 12 th batch between $0.2 T_{b}$ and $1.2 T_{b}$, or 12 booster cycles after the injection of the first batch. Bottom: Another booster cycle later. finite number of injections here. For the same reason, the ratio of final linear density to initial linear density will be slight less than $r_{d}=2.0$ given by Eq. (5.5).

The simulation is repeated with the width of the moving barrier reduced to $T_{1}=0.5 \mu \mathrm{s}$; the strength is therefore increased to $V=6.2842 \mathrm{kV}$. Because of the narrower width of the barrier, at the injection of the first batch, the left side of the barrier can be placed at $6.7 T_{b}$, correspondingly the first batch between $5.7 T_{b}$ and $6.7 T_{b}$, without particle loss. Successive batch injections are carried on in the same way. The top plot of Fig. 8 shows the situation of one booster cycle after the injection of the 12th batch at a position between $0.2 T_{b}$ and $1.2 T_{b}$, or 12 booster cycles after the injection of the first batch. Compared with the top plot of Fig. 7, all particles are now inside the designated momentum spread. Adiabatic capture can begin immediately. Thus, one booster cycle can be saved. However, the longitudinal spread is contained within $6.2 T_{b}$, which is not much less than the $\sim 6.5 T_{b}$ in the bottom plot of Fig. 7 . Thus the emittance ratio and linear-density ratio should be roughly the same as in the case of the $1 \mu$ s moving barrier.

\section{B. Discussions}

It is nontrivial to compute the initial positioning of the moving barrier relative to the position of the stationary barrier. A sufficient condition is that a particle will not be seeing the two barriers in the same revolution turn. This implies that a particle should emerge from the moving barrier before entering into the stationary barrier. Particle 2 at the lower right corner of the batch will emerge first from the moving barrier. At the critical condition, it is easy to compute using Eq. (2.3) that it takes the particle $N_{2}=3119$ turns to pass through the moving barrier of width $1.0 \mu \mathrm{s}$. At this moment, the barrier moves to the left a distance of $N_{2} \dot{T}_{2} T_{0}=0.26 T_{b}$. Thus at the injection of the first batch, the right side of the moving barrier should be $0.26 T_{b}$ to the left side of the left edge of the stationary barrier. Or, the left side of the moving barrier should be at the location $\left[7-0.26-(0.25+1.0) / T_{b}\right] T_{b}=5.95 T_{b}$ along the ring. But actually we can place it at $6.5 T_{b}$ without any particle loss. This is because we can allow the particle to interact with both barriers in the same revolution turn, provided that no particle will be energetic enough to penetrate the stationary barrier or to go outside the designated final energy spread of the beam.

Barrier waves are usually used to confine a beam so that it will stay within a designated region along the accelerator ring. The integrated sizes of the barriers need not be accurate as long as they are large enough to confine the particles of the highest and lowest energies. However, this is not true here. Although the injection process does not depend on the shape of the moving barrier, its integrated size has to be accurate in order to have the final positive and negative energy spreads be equal and opposite. Otherwise, after reflection by the stationary barrier, the positive 
energy spread will be modified, resulting possibly in an increase in the longitudinal emittance and particle loss. Here, we would like to study the effect of such an error. The top plot of Fig. 9 shows the situation when $V T_{1}$ is $10 \%$ larger than its critical value, while the width of the barrier is kept at $T_{1}=0.5 \mu \mathrm{s}$. The plot shows the moment one booster cycle after the injection of the 12th batch and should be compared with the top plot of Fig. 8. For a stronger moving barrier, most particles will emerge from the barrier at higher energies. This is evident for the particles with the least energy in the batch. We see a final negative momentum spread of $\delta_{f 2}=-1.206 \times 10^{-3}$, which is about $10 \%$ less negative than the $-1.344 \times 10^{-3}$ in the top plot of Fig. 8. Actually, the negative normalized final momentum spread is given by

$$
\bar{\delta}_{f 2}=\frac{1}{2}-\sqrt{\left(\frac{1}{2 x}+\bar{\Delta}\right)^{2}-A},
$$
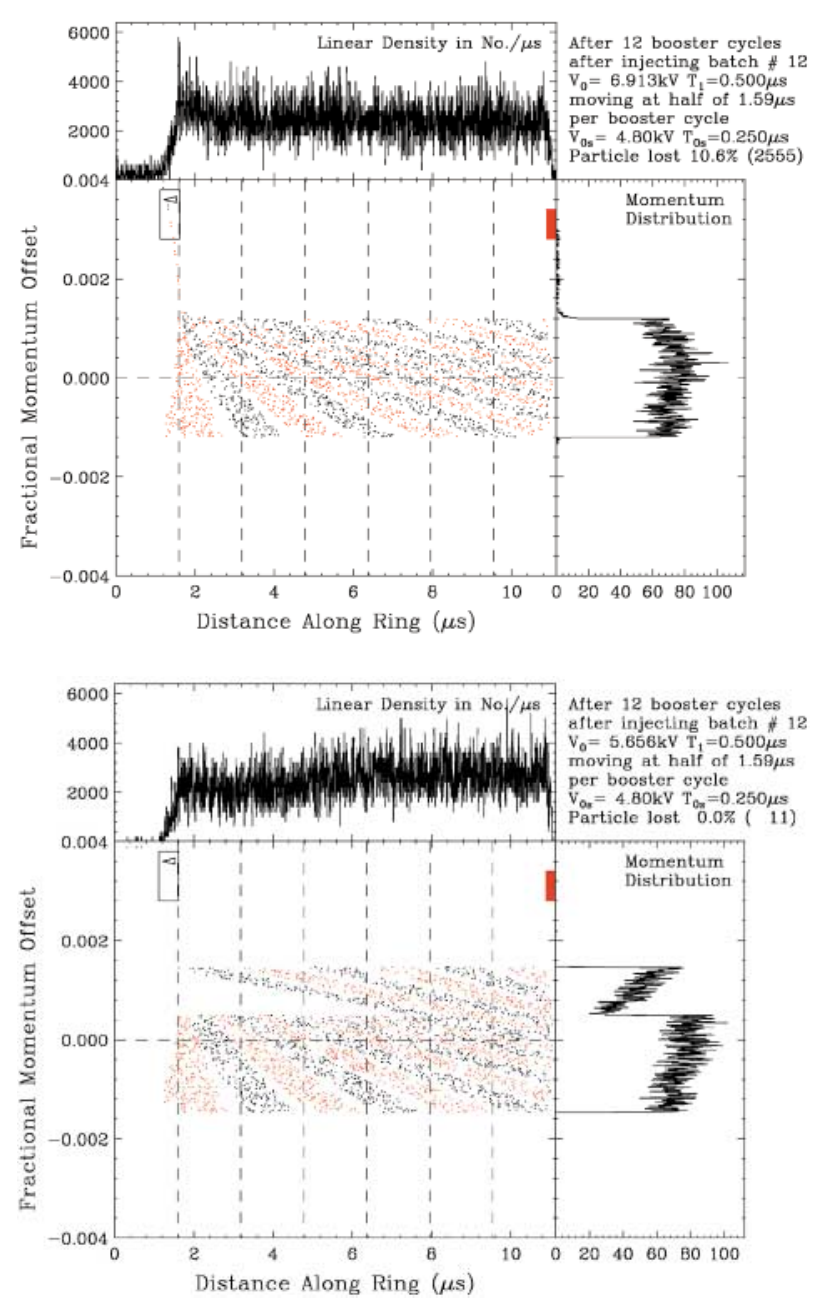

FIG. 9. (Color) Plots showing the moment one booster cycle after the injection of the 12th batch. Everything is the same as in the top plot of Fig. 8, except $V T_{1}$ of the moving barrier is increased by $10 \%$ in the top plot and decreased by $10 \%$ in the bottom plot. The width of the moving barrier is kept fixed at $T_{1}=0.5 \mu \mathrm{s}$. where the normalized initial full momentum spread is $\bar{\Delta}=$ $\sqrt{2}-1$. Here, $A$ is proportional to $V T_{1}$ and its critical value is $1 /\left(4 x^{2}\right)$. Putting in $x=\frac{1}{2}$ and $A=1.1$, we arrive at the $10 \%$ reduction as given by the simulation. For particles of the highest energies, many of them will not be able to make their way out of the enhanced moving barrier, resulting in beam loss. Some of the loss is evident in the plot at the location of the moving barrier. The total loss recorded is 2555 . There are 2000 macroparticles in each batch, giving a loss of $10.6 \%$.

The situation of a reduction of $V T_{1}$ by $10 \%$ from its critical value is shown in the bottom plot of Fig. 9. Now all particles will emerge from the moving barrier earlier and their energies are therefore reduced. According to Eq. (6.6), $\bar{\delta}_{f 2}$ will be $9.8 \%$ more negative. The simulation gives $9.2 \%$. We see that final momentum offset of particles with the highest energy is affected most. According to Eq. (5.2), $\bar{\delta}_{f 1}$ will be reduced by $63 \%$, which agrees with the result of the simulation. However, the final positive momentum spread is determined by the negative final momentum spread after the reflection by the stationary barrier. Thus, the final momentum spread actually increases by $9.8 \%$, about the amount of the error in $V T_{1}$. The loss recorded is 11 , or $0.045 \%$. This loss comes from the reflection by the stationary barrier. Some of these particles have energies high enough that their left-drift velocities are higher than that of the moving barrier. Eventually, some of these particles catch up with the moving barrier and get lost.

This injection method requires only two barriers, one moving and one stationary. Unfortunately, they are of the same sign. In practice, one must also introduce barriers of the opposite sign to cancel the charge accumulation. Therefore, space along the ring must be available for their introduction. This may shorten the space available for successive batch injections. Therefore, we may require narrow barriers (less than $0.5 \mu \mathrm{s})$ with high voltages $(\gtrsim 6 \mathrm{kV})$ to ensure the injection of 12 booster batches.

\section{Adiabatic capture}

We want to examine the adiabatic capture of a coasting beam in the Main Injector at injection. Here, we set the criterion that the relative change in bucket height or bucket area $A_{b}$ should be much slower than the synchrotron angular frequency $\omega_{s}$, or

$$
\omega_{s} \gg \frac{1}{A_{b}} \frac{d A_{b}}{d t} .
$$

Bunch area is proportional to $\sqrt{V_{\mathrm{rf}}}$ and $\omega_{s}$ is also proportional to $\sqrt{V_{\mathrm{rf}}}$, where $V_{\mathrm{rf}}$ is the rf voltage. Let $a=$ $\omega_{s} / \sqrt{V_{\text {rf }}}$. Then

$$
a \gg \frac{1}{2 V_{\mathrm{rf}}^{3 / 2}} \frac{d V}{d t},
$$




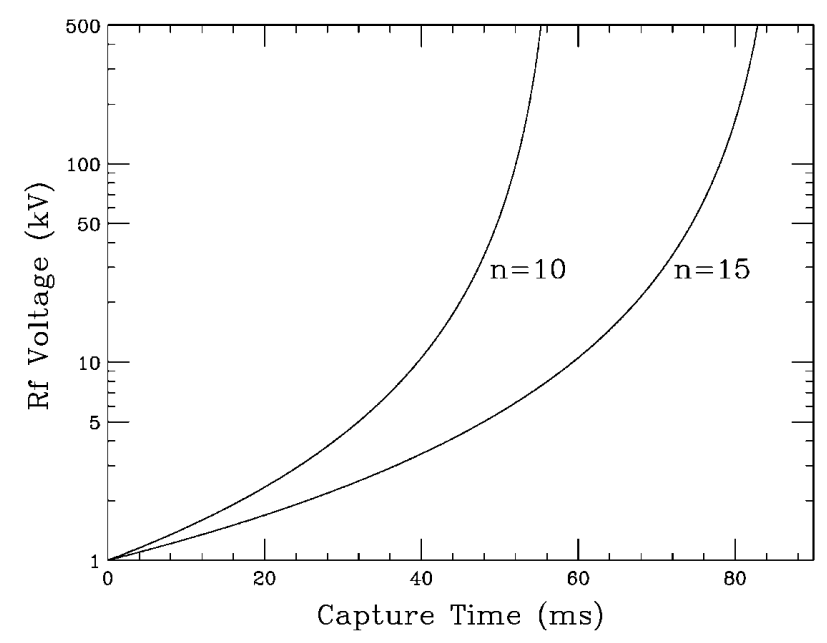

FIG. 10. Plot of capture time in ms for various initial and final rf voltages $V_{1}$ and $V_{2}=500 \mathrm{kV}$. The relative rate of change of the bucket area is taken as $1 / n$ of the angular synchrotron frequency, where $n=10$ or 15 .

or

$$
a t \gg \frac{1}{\sqrt{V_{\mathrm{rf}}(t)}}+C
$$
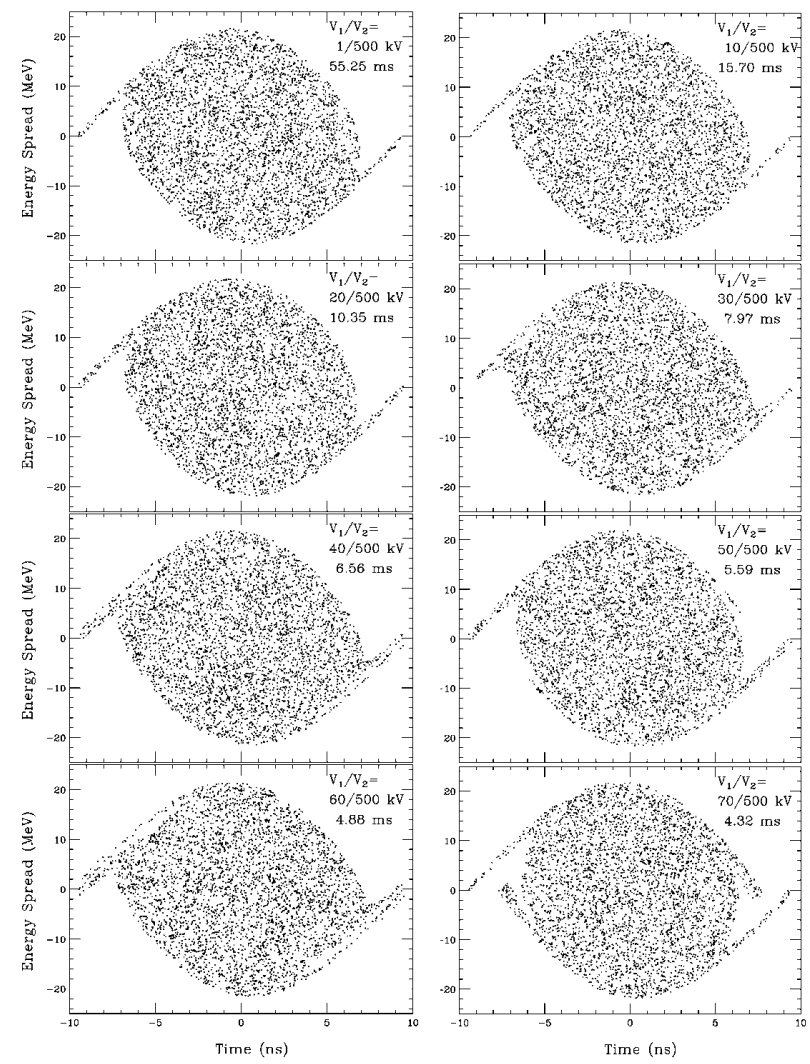

FIG. 11. Plot of a $53 \mathrm{MHz}$ bunch in the longitudinal phase space after adiabatic capture starting from various initial rf voltage $V_{1}$ to the final $V_{2}=500 \mathrm{kV}$. Adiabaticity is fixed at $n=10$ and the initial bunch area is $2 \times 12 \times$ $11.134 / 588=0.4544 \mathrm{eVs}$. At $V_{2}=500 \mathrm{kV}$, the final half bunch height is $21.65 \mathrm{MeV}$ and half width $7.28 \mathrm{~ns}$ when matched to the rf bucket. where $C$ is a constant. We cannot start from $V_{\text {rf }}=0$, because the constant $C$ will become infinite. So we start from $V_{\mathrm{rf}}=V_{1}$ at $t=0$ and end at $V_{\mathrm{rf}}=V_{2}$ at $t=t_{2}$, giving $C=1 / \sqrt{V_{1}}$. Define $\omega_{s 1}$ as the initial angular synchrotron frequency (at $V_{\mathrm{rf}}=V_{1}$ ). Then

$$
\omega_{s 1} t \gg \sqrt{\frac{V_{1}}{V(t)}}+1 \text {. }
$$

If good adiabaticity requires the relative rate of change of the bucket area to be $1 / n$ of the synchrotron angular frequency, we obtain the rf voltage curve

$$
\sqrt{\frac{V_{\mathrm{rf}}(t)}{V_{1}}}=\frac{1}{1-\omega_{s 1} t / n} .
$$

For example, if we start from $V_{1}=5 \mathrm{kV}$ and end at $V_{2}=$ $500 \mathrm{kV}$, the capture time will be $t_{2}=23.28 \mathrm{~ms}$ when $n=10$.

In the injection scheme discussed here, if the injected booster batch has a half energy spread of $5 \mathrm{MeV}$, after exiting the moving barrier, the half spread becomes $12 \mathrm{MeV}$. We want to capture the beam into buckets with rf harmonic $h=588$. There are three variables: $V_{1}, V_{2}$, and $n$. It appears that $n$ has to be larger than 10 . In
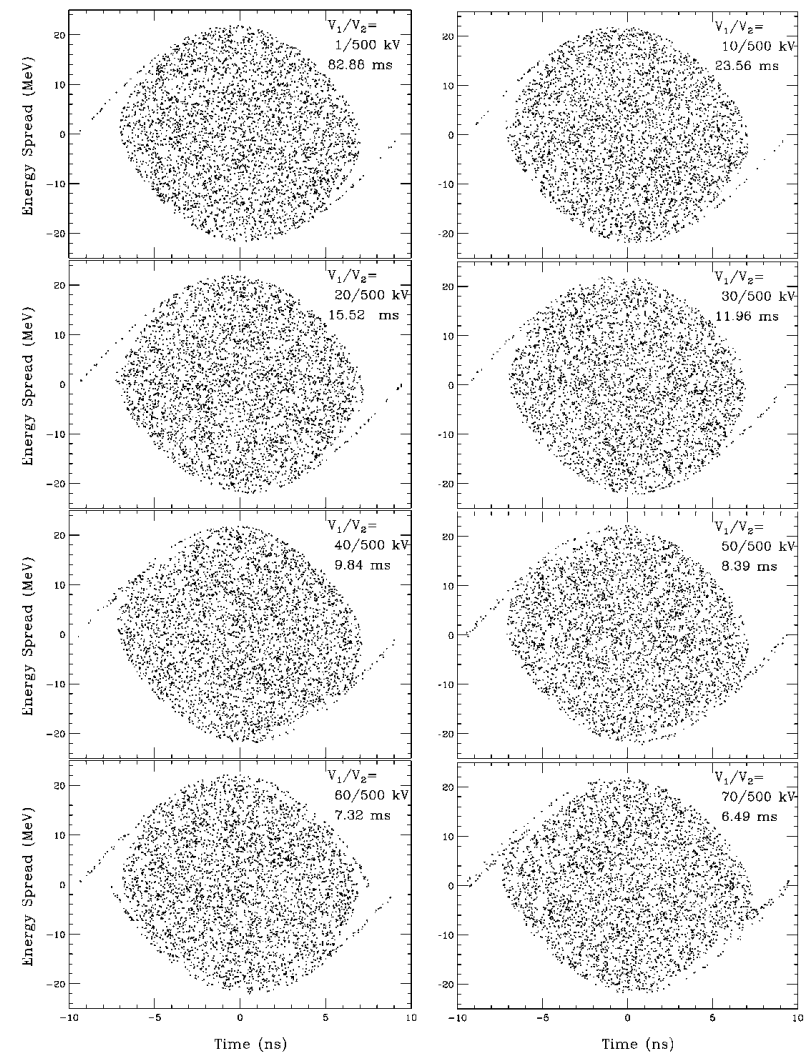

FIG. 12. Plot of a $53 \mathrm{MHz}$ bunch in the longitudinal phase space after adiabatic capture starting from various initial rf voltage $V_{1}$ to the final $V_{2}=500 \mathrm{kV}$. Adiabaticity is fixed at $n=15$ and the initial bunch area is $2 \times 12 \times 11.134 / 588=$ $0.4544 \mathrm{eV} \mathrm{s}$. At $V_{2}=500 \mathrm{kV}$, the final half bunch height is 21.65 MeV and half width $7.28 \mathrm{~ns}$ when matched to the rf bucket. 
fact, $n=15$ is a more appropriate number. The final $\mathrm{rf}$ voltage cannot be too small because the initial half energy spread is $12 \mathrm{MeV}$ already. The initial bunch area is $2 \Delta E_{f 1} T_{0} / h=0.4544 \mathrm{eV} \mathrm{s}$. For such a bucket area, a rf voltage of $V_{\text {rf }}=268.3 \mathrm{kV}$ is required. For this reason, we set $V_{2}=500 \mathrm{kV}$. This rf voltage will establish a bucket with area $0.620 \mathrm{eV} \mathrm{s}$ and half height $23.16 \mathrm{MeV}$. On the other hand, a bunch of area $0.4544 \mathrm{eV} \mathrm{s}$ will have half length $7.28 \mathrm{~ns}$ and half height $21.65 \mathrm{MeV}$. Thus, $V_{2}$ cannot be much less than $500 \mathrm{kV}$. Then, $V_{1}$ becomes the only variable. The capture time for a given $V_{1}$ and $V_{2}$ can be read off easily from Fig. 10. As an example,
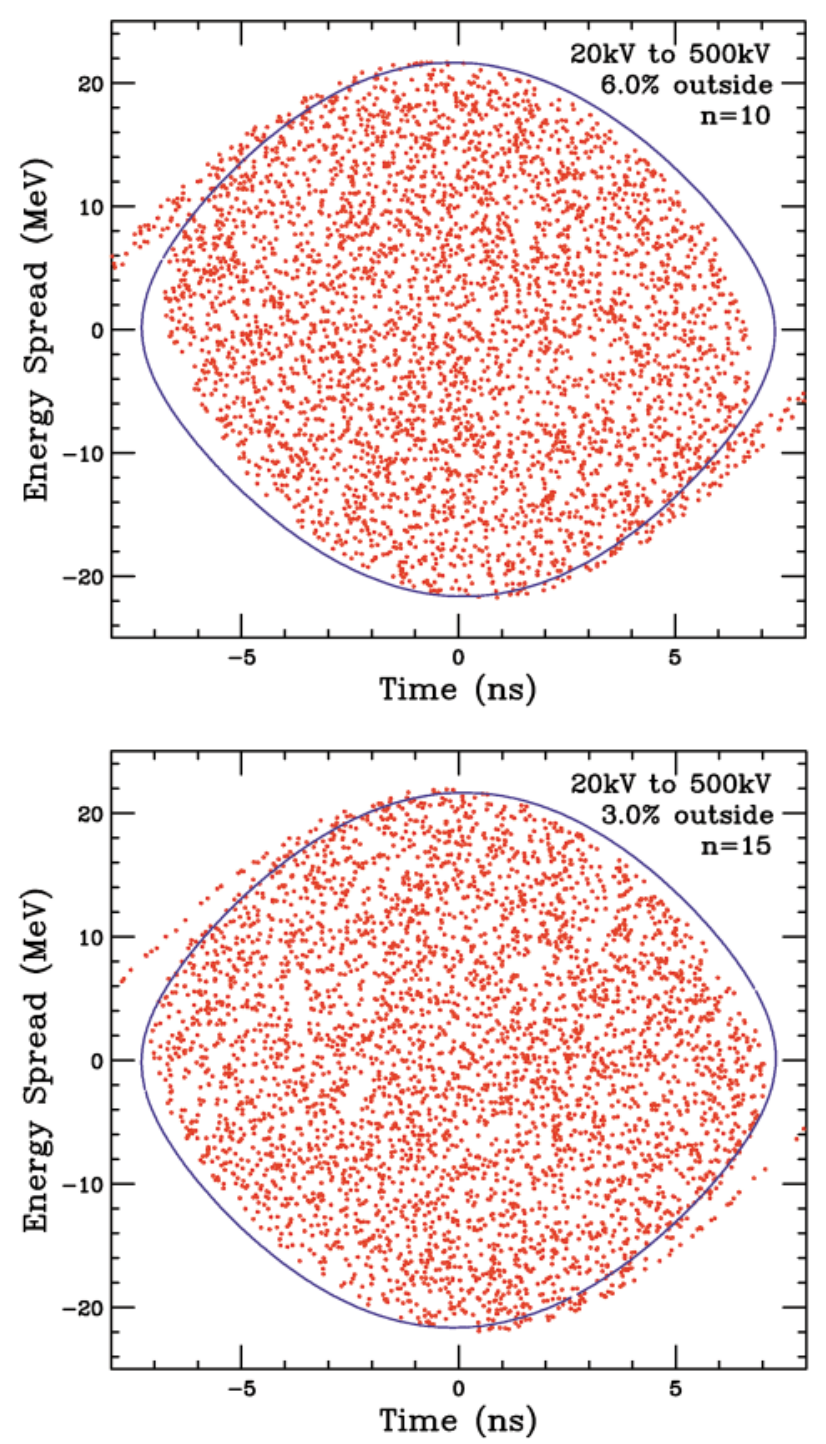

FIG. 13. (Color) Adiabatic capture starting from $V_{1}=20 \mathrm{kV}$ to $V_{2}=500 \mathrm{kV}$ with adiabaticity $n=10$ in the top plot and $n=15$ in the bottom plot. The solid blue curve in each plot represents the boundary of the bunch of $0.4544 \mathrm{eV} \mathrm{s}$ (initial area) matched to the $\mathrm{rf}$ voltage of $V_{2}$. Particles outside the curve may be lost eventually. The loss is $6.0 \%$ for $n=10$ and reduces to $3.0 \%$ at $n=15$.
$V_{1}=20 \mathrm{kV}, V_{2}=500 \mathrm{kV}$, and $n=15$ leads to a capture time of $15.52 \mathrm{~ms}$ or 1393 turns. Changing $n$ to 10 will reduce the capture time to $10.35 \mathrm{~ms}$ or 929 turns.

Simulations of adiabatic capture are performed from various initial rf voltage $V_{1}$ to the final $V_{2}=500 \mathrm{kV}$. The results are shown in Fig. 11 for the adiabaticity $n=10$ and in Fig. 12 for the adiabaticity $n=15$. It appears that the capture is acceptable even if we start with rf voltage $V_{1}=50 \mathrm{kV}$. The capture time will be $5.6 \mathrm{~ms}$ at $n=10$ and $8.4 \mathrm{~ms}$ at $n=15$. In Fig. 13, we give a closer look at the captures again from $V_{1}=20 \mathrm{kV}$ to $V_{2}=500 \mathrm{kV}$ at adiabaticity $n=10$ in the top plot and $n=15$ in the bottom plot. We also superimpose as solid blue curves the boundaries of the bunches with area $0.4544 \mathrm{eV} \mathrm{s}$ that are matched to the rf voltage of $V_{2}=500 \mathrm{kV}$. Particles outside the blue curves are considered lost eventually. Here, we see a $6.0 \%$ loss for $n=10$ and only $3.0 \%$ loss for $n=15$. We can conclude that an adiabatic capture time of $\sim 10 \mathrm{~ms}$ will be quite adequate using $n=15$.

\section{BEAM LOADING}

Because of the high beam current, beam loading voltage induced in the Main Injector rf cavities can become a serious problem. In fact, beam loading represents the most serious problem in slip stacking. A bunch at the $53 \mathrm{MHz}$ rf voltage (harmonic $h=588$ ) containing $6.0 \times 10^{10}$ protons carries a charge of $q=9.61 \mathrm{nC}$. If the bunch is short, it can be approximated by a macroparticle, which will induce a total instantaneous beam loading voltage $V_{b 0}=$ $q N_{c} \omega_{r} R_{L} / Q_{L}=5.5 \mathrm{kV}$ across the gaps of $N_{c}=18$ cavities, where $R_{L}=500 \mathrm{k} \Omega, Q_{L}=5000$, and $\omega_{r} /(2 \pi)=$ $52.7 \mathrm{MHz}$ are, respectively, the loaded shunt impedance, loaded quality factor, and resonant frequency of each cavity. For a batch of 84 such bunches injected into the Main Injector, a beam loading voltage of $5.97 \mathrm{kV}$ is induced in the $\mathrm{rf}$ cavities at the passage of the first bunch. This beam loading voltage increases to $485 \mathrm{kV}$ after the passage of the last bunch. Thus the difference in beam loading voltages experienced by the last and first bunches is $479 \mathrm{kV}$. Taking into account of the $28.3 \mathrm{~ns}$ half lengths of the bunches in a parabolic distribution, this difference becomes $388 \mathrm{kV}$ when steady state is reached. During normal operation, the total rf voltage at injection is 1.2 MV. If the designed synchronous phase $\phi_{s}=0$ is synchronized to the middle bunch of the batch, the rf phase errors introduced become $\Delta \phi_{s}= \pm 9.18^{\circ}$ for the first and last bunches. Eventually, the longitudinal emittances of the bunches will be increased up to $18 \%$, which is considered still tolerable.

During slip stacking, although the bunch density is doubled, the beam loading voltage may not increase at all. This is because in order to allow two series of buckets to staywithin the momentum aperture of the ring, the bucket height of each series must be reduced to a minimum and the bunches are therefore lengthened to a size that almost fills up the buckets [11]. As a result, the rf voltage must be kept 
at the low value of $\sim 64 \mathrm{kV}$, which is almost 7 times less than the difference in beam loading voltages seen by the first and last bunches. The rf phase errors of the bunches now become so large that most beam particles will be driven out of the buckets. The following methods have been proposed to control the beam loading voltage in slip stacking [12]:

(i) Tuning all cavities to the nominal $8 \mathrm{GeV}$ frequency.

(ii) Using only two or four of the cavities to produce the required rf voltage and deQing the remaining cavities. One simple technique that may deQ the cavities by a factor of 3 is to turn off the screen voltage to reduce the tube plate resistance.

(iii) Feed forward the signal of the wall current monitored at a resistive-wall gap to the cavity drivers. Experience at the former Fermilab Main Ring expects to achieve a tenfold reduction in the effective wall current flowing into the cavities.

(iv) Feedback on all the cavities. A signal proportional to the gap voltage is amplified, inverted, and applied to the driver amplifier. Based on experience in the Main Ring and results achieved elsewhere, a 100-fold reduction can be achieved.

In the present continuous multiple injection scheme using barriers, the booster bunches contained in the batches before injection will be made very long in the booster so that the momentum spread can be reduced. These bunches start to debunch immediately after being injected into the Main Injector. For a completely uniformly distributed beam, there is no $53 \mathrm{MHz}$ rf component and therefore no beam loading at all. Here, although the distribution is not uniform (see, for example, Fig. 8), the $53 \mathrm{MHz}$ rf component of the beam current at any moment of the injection is found to be less than $0.25 \%$ of twice the dc beam current, implying that the beam loading voltage will be reduced at least 400 times.

Actually no $\mathrm{rf}$ voltage is required in this injection scheme. There are two controls in the Main Injector rf system. The 18 cavities can be separated into two groups. As a result, the gap voltage in each cavity need not be small at all, because counterphasing can be employed to arrive at zero rf voltage with the tiny beam loading voltage taken into account. On the other hand, counterphasing cannot be performed in slip stacking in order to avoid low gap voltages, because the two rf controls have already been used in generating the two series of buckets of slightly different $\mathrm{rf}$ frequencies.

Of course, counterphasing of 18 cavities may not be the best method in this continuous injection scheme, because it is very difficult to control the phases of the cavities in order to arrive at exactly zero rf voltage. Another method is to turn off the rf drive and mechanically short the cavity gaps. The Main Injector is presently equipped with fast mechanical shorts, which can be inserted in $100 \mathrm{~ms}$ and removed in $50 \mathrm{~ms}$ [13]. The ideal method may be a combination of both counterphasing and mechanical shorts. First, the rf drive of 16 cavities is turned off and shorts are inserted. Second, counterphasing is used for the two remaining cavities to arrive at zero rf voltage with the consideration of the tiny beam loading voltage included. Further fine adjustment can be implemented using a fast low-level feedback.

\section{CONCLUSION}

We discuss in detail a method to double the linear beam density of the Fermilab Main Injector using barrier waves. The injection is continuous and no extra time is required except for the final adiabatic capture, which can be accomplished in about $10 \mathrm{~ms}$. In contrast to slip stacking, rf manipulations of intense beams at very low cavity gap voltages can be avoided and beam loading does not appear to be a problem.

\section{ACKNOWLEDGMENTS}

The author wishes to thank Dr. D. Wildman for several very meaningful discussions on beam loading. This work was supported by the U.S. Department of Energy under Contract No. DE-AC02-76CH03000.

\section{APPENDIX}

In this appendix, we are going to show that the injection process discussed in this paper depends only on the integrated barrier voltage and does not depend on the shape of the barrier. Thus, whenever $V T_{1}$ appears, we can make the substitution

$$
V T_{1}=\int V(\tau) d \tau
$$

where $V(\tau)$ is a general barrier wave.

The Hamiltonian describing the longitudinal motion of a particle of charge $e$ interacting with a stationary barrier wave can be written as

$$
H=-\frac{1}{2}|\eta| \delta^{2}-\frac{e}{\beta^{2} E T_{0}} \int_{0}^{\tau} V\left(\tau^{\prime}\right) d \tau^{\prime},
$$

so that the equations of motion are

$$
\begin{aligned}
& \frac{d \tau}{d t}=-|\eta| \delta, \\
& \frac{d \delta}{d t}=\frac{e V(\tau)}{\beta^{2} E T_{0}} .
\end{aligned}
$$

Here, $\delta$ and $\tau$ are, respectively, the fractional momentum offset and arrival time behind an on-momentum particle. ${ }^{4}$ For simplicity, we have also restricted ourselves to operation below transition.

\footnotetext{
${ }^{4}$ Time lag is chosen here so that positive $\tau$ points to the right in Fig. 2 and Figs. 6-9, and this is the same direction the integration over the barrier voltage is performed in the Hamiltonian in Eq. (A.2).
} 
The particle has initial fractional momentum offset $\delta_{i 1}$ at time $t=0$ when it starts entering the barrier. Then from the Hamiltonian

$$
-\frac{1}{2}|\eta| \delta_{i 1}^{2}=-\frac{1}{2}|\eta| \delta^{2}(\tau)-\frac{e}{\beta^{2} E T_{0}} \int_{0}^{\tau} V\left(\tau^{\prime}\right) d \tau^{\prime} .
$$

We can also start from the equations of motion,

$$
\frac{d}{d t} \frac{d \tau}{d t}=-\frac{|\eta| e V(\tau)}{\beta^{2} E T_{0}} .
$$

Multiplying by $2 d \tau / d t$,

$$
\begin{gathered}
\frac{d}{d t}\left(\frac{d \tau}{d t}\right)^{2}=-\frac{2|\eta| e V(\tau)}{\beta^{2} E T_{0}} \frac{d \tau}{d t} \\
\left(\frac{d \tau}{d t}\right)^{2}=\left(\frac{d \tau}{d t}\right)_{i 1}^{2}-\int_{0}^{\tau} \frac{2|\eta| e V\left(\tau^{\prime}\right)}{\beta^{2} E T_{0}} d \tau^{\prime} .
\end{gathered}
$$

Substituting the phase equation, we obtain again

$$
\delta^{2}(\tau)=\delta_{i 1}^{2}-\int_{0}^{\tau} \frac{2 e V\left(\tau^{\prime}\right)}{|\eta| \beta^{2} E T_{0}} d \tau^{\prime} .
$$

Now the barrier is moving at the rate $\dot{T}_{2}$ to the left. When the particle is at time lag $\tau$, it is seeing the barrier wave at time lag $\tilde{\tau}=\tau+\dot{T}_{2} t$. The Hamiltonian changes to

$$
H=-\frac{1}{2}|\eta| \delta^{2}-\frac{e}{\beta^{2} E T_{0}} \int_{0}^{\tau+\dot{T}_{2} t} V\left(\tau^{\prime}\right) d \tau^{\prime},
$$

and the energy equation becomes

$$
\frac{d \delta}{d t}=\frac{e V\left(\tau+\dot{T}_{2} t\right)}{\beta^{2} E T_{0}}
$$

Let us go to the rest frame of the barrier. The phase lag for the particle becomes $\tilde{\tau}$ and the phase equation is

$$
\frac{d \tilde{\tau}}{d t}=\frac{d \tau}{d t}+\dot{T}_{2}=-|\eta|\left(\delta-\frac{\dot{T}_{2}}{|\eta|}\right)
$$

We can write the energy equation as

$$
\frac{d}{d t}\left(\delta-\frac{\dot{T}_{2}}{|\eta|}\right)=\frac{e V(\tilde{\tau})}{\beta^{2} E T_{0}} .
$$

In other words, in the rest frame of the moving barrier, the Hamiltonian becomes

$$
H=-\frac{1}{2}|\eta| \tilde{\delta}^{2}-\frac{e}{\beta^{2} E T_{0}} \int_{0}^{\tilde{\tau}} V\left(\tau^{\prime}\right) d \tau^{\prime},
$$

where

$$
\begin{aligned}
& \tilde{\tau}=\tau+\dot{T}_{2} t, \\
& \tilde{\delta}=\delta-\frac{\dot{T}_{2}}{|\eta|}
\end{aligned}
$$

are the new canonical variables. We therefore have the solution

$$
\begin{aligned}
\delta_{f 1} & -\frac{\dot{T}_{2}}{|\eta|} \\
& =-\sqrt{\left(\delta_{i 1}-\frac{\dot{T}_{2}}{|\eta|}\right)^{2}-\frac{2}{|\eta| T_{0} \beta^{2} E} \int_{0}^{\tau_{f}} V\left(\tau^{\prime}\right) d \tau^{\prime}},
\end{aligned}
$$

where $\tau_{f}$ is the total width of the barrier and $\delta_{f 1}$ is the fractional momentum offset of the particle on exiting the barrier. Here, the negative sign in front of the square root sign has been chosen, because as $\tau_{f} \rightarrow 0$, one must have $\delta_{f 1} \rightarrow \delta_{i 1}$, noting that $\delta_{i 1}<0$. Equation (2.7) is just a special case of Eq. (A.16) when the barrier is of a square shape. Starting from Eq. (2.7), we see that $V T_{1}$ comes about as one variable. Thus, the replacement in Eq. (A.1) can be made in all the equations that follow.

[1] Fermilab Report, "Run II Handbook," http://wwwbd.fnal.gov/runII/index.html.

[2] Fermilab Report, "Plan for Tevatron Run IIb," http://cosmo.fnal.gov/run2b/Documents/TDR/tdr.pdf

[3] C. Ankenbrandt, Fermilab Report No. FN-352, 1981; F. E. Mills, BNL Internal Report No. AADD 176, 1971.

[4] D. Boussard and Y. Miszumachi, IEEE Trans. Nucl. Sci. 26, 3623 (1979); J. P. Delahaye, P. Lefevre, and J. P. Riunaud, IEEE Trans. Nucl. Sci. 26, 3565 (1979).

[5] J. A. MacLachlan, Fermilab Report No. FN-0711, 2001.

[6] S. Machida (private communication).

[7] K. Y. Ng, in Proceedings of the 1997 Particle Accelerator Conference, Vancouver, B.C., Canada, 1997, (IEEE, Piscataway, NJ, 1998), p. 1003; Fermilab Report No. FN-654, 1997

[8] M. Blaskiewicz, J. M. Brennan, T. Roser, K. Smith, R. Spitz, A. Zaltsmann, M. Fujieda, Y. Iwashita, A. Noda, M. Yoshii, Y. Mori, C. Ohmori, and Y. Sato, in Proceedings of the 1999 Particle Accelerator Conference, New York, 1999 (IEEE, Piscataway, NJ, 1999), p. 2280; M. Fujieda, Y. Iwashita, A. Noda, Y. Mori, C. Ohmori, Y. Sato, M. Yoshii, M. Blaskiewicz, J. M. Brennan, T. Roser, K. Smith, R. Spitz, and A. Zaltsmann, ibid., p. 857.

[9] J. Griffin, Fermilab Internal Report, "Momentum Stacking in the Main Injector using Longitudinal Barriers," 1996.

[10] J. Griffin (private communication).

[11] Compared with a point bunch, the beam loading voltage induced by a bunch filling the whole bucket uniformly is smaller by a factor of 3 . In other words, the ratio of the beam current component at $\mathrm{rf}$ frequency to twice the dc beam current is $\frac{1}{3}$.

[12] Shekhar Shukla, John Marriner, and James Griffin, in Proceedings of the 1996 DPF/DPB Summer Study on New Directions for High-Energy Physics, Snowmass, CO, 1996 (Stanford Linear Accelerator Center, Stanford, CA, 1997), p. 150.

[13] D. Wildman, in Proceedings of the 1991 IEEE Particle Accelerator Conference, San Francisco, 1991 (IEEE, Piscataway, NJ, 1991), p. 410. 